\title{
Análise dos Fatores de Risco Para Desenvolvimento de Encefalopatia Crônica Não Progressiva
}

\section{Analysis of Risk Factors for Chronic Encephalopathy Development of Non-Phase}

\author{
LUCIANA SOUSA MATA FERREIRA ${ }^{1}$ \\ VANDIEL BARBOSA SANTOS ${ }^{2}$ \\ PRISCILA BARROS PESSOA ${ }^{2}$ \\ POLLYANNA SOUSADA SILVA ${ }^{2}$ \\ SURAMA MARIA BANDEIRA DE SOUSA ${ }^{3}$ \\ LEANDRO MARQUES SILVA ${ }^{4}$
}

\section{RESUMO}

Introdução: A encefalopatia crônica não progressiva (ECNP) é uma disfunção sensório- motora, que afeta o sistema nervoso central no momento da sua maturação estrutural e funcional. Estudos revelam que o contexto socioeconômico das famílias podem influenciar na ocorrência dos casos de ECNP. Objetivo: Analisar os fatores de riscos para desenvolvimento de ECNP. Material e Método: Trata-se de uma pesquisa quali-quantitativa, transversal, de caráter descritivo. Foram incluídas na pesquisa crianças com diagnóstico médico de ECNP, em atendimento multidisciplinar nas instituições escolhidas, procedentes de qualquer localidade, do estado do Piauí, e de ambos os gêneros. Foram extraídas dos prontuários das crianças com ECNP, informações quanto ao histórico pré-concepcional, gestacional, parto e dados da criança. Resultados: Foram analisadas 30 crianças, com média de idade de 7,31 anos, sendo que $56,7 \%$ eram do gênero feminino. A idade média das mães no período gestacional foi 23 anos, sendo a gravidez desejada em $56,7 \%$ dos casos. Cerca de $13,3 \%$ relataram ter utilizado medicamentos abortivos e $6,7 \%$ ter consumido drogas ilícitas ou ingerido bebidas alcoólicas, $10 \%$ delas eram tabagistas e $43,3 \%$ realizaram o parto normal. Conclusão: O histórico pré-concepcional e determinados hábitos maternos adotados durante a gravidez interferem na formação do sistema nervoso central, e assim, contribuem para o desenvolvimento da ECNP.

\section{DESCRITORES}

Fatores de risco. Paralisia Cerebral. Crianças.

\begin{abstract}
Introduction: The non-progressive chronic encephalopathy (ECNP) is a sensorimotor disorder that affects the central nervous system at the time of its structural and functional maturation. The studies show that the socioeconomic background of families may influence the occurrence of cases of ECNP. Objective: To analyze the risk factors for developing ECNP. Methods: This is a qualitative and quantitative research, cross-sectional, descriptive character. They were included in the study children with a diagnosis of ECNP, in multidisciplinary care in the chosen institutions, coming from any location, the state of Piauí, and both genders. Children were extracted with records of ECNP, information on the history preconception, pregnancy, childbirth and child data. Results: 30 children were analyzed, with a mean age of 7.31 years, and $56.7 \%$ were female. The average age of mothers during pregnancy was 23 , the desired pregnancy and in $56.7 \%$ of cases. About $13.3 \%$ reported having used abortifacient drugs and $6.7 \%$ having used illicit drugs or consumed alcohol, $10 \%$ of them were smokers and $43.3 \%$ had normal birth. Conclusion: The preconception certain maternal history and habits adopted during pregnancy interfere with the formation of the central nervous system and thus contribute to the development of ECNP.
\end{abstract}

\section{DESCRIPTORS}

Risk factors. Cerebral Palsy. Children.

\footnotetext{
Fisioterapeuta, Teresina (PI), Brasil.

Enfermeiro do programa de Residência Multiprofissional da Universidade Federal do Maranhão- UFMA, São Luís (MA), Brasil.

Supervisora do programa de Residência Multiprofissional da Universidade Federal do Maranhão- UFMA, São Luís (MA), Brasil.

Fisioterapeuta da UTI Pediátrica do Hospital da Criança Dr. Odorico de Amaral Matos- HC, São Luís-MA, Brasil.
} 
$\mathrm{A}$ encefalopatia crônica não progressiva (ECNP), também conhecida como paralisia cerebral (PC), é consequência de uma lesão estática, que afeta o sistema nervoso central (SNC) em fase de maturação estrutural e funcional. Trata-se de uma disfunção predominantemente sensório-motora, que envolve distúrbios no tônus muscular, postura e movimentação voluntária, resultando a longo prazo em deformidades ósseas e déficits funcionais ${ }^{1}$.

A incidência de ECNP nos Estados Unidos oscila entre 1,5 e 5,9/1.000 nascidos vivos, segundo dados de 2008. No Brasil, não existem dados estatísticos fidedignos, estima-se que a cada mil crianças que nascem, sete são acometidas por esta patologia. Nos países em desenvolvimento, esse número pode estar relacionado às intercorrências gestacionais, atendimentos médico insatisfatório e condições precárias de nutrição materna e infantil ${ }^{2}$.

É importante ressaltar que a etiologia da ECNP é motivo de investigação entre pesquisadores de diversos países. Atualmente, foram encontrados vários fatores que interagem entre si, sugerindo que seja uma doença multifatorial. Os fatores determinantes podem ser pré-natais (más-formações genéticas, infecções maternas), perinatais (anóxia neonatal, prematuridade e baixo peso ao nascer) e pós-natais (meningites, encefalites e vasculites cerebrais) ${ }^{3}$.

As precárias condições de assistência no período gestacional, parto e período neonatal, expressam os recursos técnicos limitados para a população de baixa renda. Esse plano de fundo, paradoxal, expõe as desigualdades sociais persistentes na sociedade brasileira penalizando aqueles/as que certamente mais necessitam de atenção e cuidados ${ }^{4}$. Dessa forma, a prevenção tem sido considerada a principal medida no que diz respeito à qualidade do atendimento prestado à mulher e ao recém-nascido, a fim de evitar a ocorrência de eventos indesejados, tais como afecções durante a gestação e complicações durante o trabalho de parto 5 .

$\mathrm{O}$ processo de atenção à gestante não se limita ao pré-natal, uma vez que os fatores de risco, como os socioeconômicos, podem interferir no desenvolvimento normal do concepto, contudo, durante o pré-natal, os serviços de saúde têm pouco ou nenhum conhecimento acerca destas informações ${ }^{6}$.

O conhecimento prévio do contexto socioeconômico destas mães, vitimadas pela vulnerabilidade social e demográfica, fornece informações importantes sobre possíveis fatores causais, que permitem o planejamento de estratégias de padronização dos cuidados no período gestacional, visando a melhoria do atendimento prestado a estas e consequentemente a redução dos casos de ECNP, através das políticas públicas.

O objetivo do presente estudo foi analisar os fatores de riscos para desenvolvimento de encefalopatia crônica não progressiva.

\section{MATERIAL E MÉTODOS}

Trata-se de uma pesquisa de campo com abordagem descritiva, de corte transversal, caráter exploratório e natureza quali-quantitativa. $\mathrm{O}$ estudo foi submetido à análise da Comissão de Ética e Pesquisa do Centro de Ensino Unificado de Teresina (CEUT) e aprovado sob protocolo de número 09737/2012. A coleta de dados se desenvolveu em conformidade com a Resolução nº 422/12 do Conselho Nacional de Saúde que rege pesquisa envolvendo seres humanos. Após a escolha dos participantes foi solicitado através de um Termo de Consentimento Livre e Esclarecido (TCLE), a autorização dos pais ou responsáveis das crianças selecionadas para a participação destas no estudo.

O estudo foi desenvolvido em três instituições filantrópicas. Estas instituições foram selecionadas por serem referências no atendimento de crianças com comprometimento neurológico. A seleção das crianças participantes do estudo seguiu critérios não probabilísticos. Sendo incluídas na pesquisa crianças com diagnóstico médico de ECNP, em atendimento multidisciplinar nas instituições escolhidas, procedentes de qualquer localidade, do estado do Piauí e de ambos os gêneros. Foram excluídas da presente pesquisa, crianças que apresentavam alguma outra patologia de base além da ECNP, que continham dados do prontuário incompletos ou quando os pais não aceitavam a participação destas no estudo

Foram realizadas análises dos prontuários, utilizando-se de um formulário, criado com o objetivo de padronizar a coleta de informações. Neste formulário estavam contidos os dados do histórico préconcepcional, histórico gestacional, hábitos maternos nocivos, realização de consultas pré-natais e o histórico do parto; idade, gênero e diagnóstico clínico da criança.

Os dados coletados foram transferidos para um banco de dados criado em planilha do Excel, versão Microsoft Windows 7, em seguida foram submetidos à análise estatística por meio do programa Statistical Package for the Social Sciences (SPSS) versão 18.0. O nível de significância adotado foi de $\mathrm{p}=0,05$ e intervalo de confiança (IC) de 95\%. Os dados foram descritos através de médias e desvios padrão para variáveis contínuas, frequência absoluta e percentual para variáveis categóricas. Para verificar a associação entre as variáveis socioeconômicas e clínicas foi utilizado o teste qui-quadrado. 


\section{RESULTADOS}

A amostra desse estudo foi composta por trinta pacientes com ECNP. Desse total, dezessete $(56,7 \%)$ eram do sexo feminino e treze $(43,3 \%)$ do sexo masculino, com média de idade de 7,31 $\pm 3,64$ anos.

Quanto ao histórico pré-concepcional das crianças, observa-se na Tabela 1 que a idade das mães variou de 13 a 34 anos, 29 (50\%) mães da amostra eram multigestas e apenas uma mãe era primigesta (3,34\%). A gravidez foi desejada em $56,7 \%$ das mães, e $50 \%$ relataram gestações anteriores $\left(C^{2}=0,8526\right)$ com variações entre 1 a 6 gestações e $10(33,3 \%)$ mulheres relataram abortos prévios. Com relação ao histórico familiar da doença, obteve-se $\mathrm{C}^{2}=0,0053$.

O uso de medicamentos abortivos, segundo a tabela 2 , foi relatado por $13,3 \%$ das entrevistadas, cerca de $6,7 \%$ consumiram drogas ilícitas ou ingeriram bebidas alcoólicas; $10 \%$ delas são tabagistas e $90 \%$ relataram ter realizado consultas pré-natais.

A Tabela 3 apresenta o tipo e o local do parto ocorrido com as crianças com ECNP, de forma que o parto natural ocorreu em $43,3 \%$, seguido de $33,3 \%$ por via cesariana e 23,3\% por fórceps. Quanto aos locais de realização do parto, observa-se que a maioria, 96,7\% ocorreu em ambiente hospitalar e 3,3\% em ambiente domiciliar.

Demonstra-se na Figura 1 as frequências relativas quanto ao nível de instrução das mães de crianças com ECNP.

\section{DISCUSSÃO}

A predominância do gênero feminino nesta pesquisa discorda de um trabalho realizado, na cidade

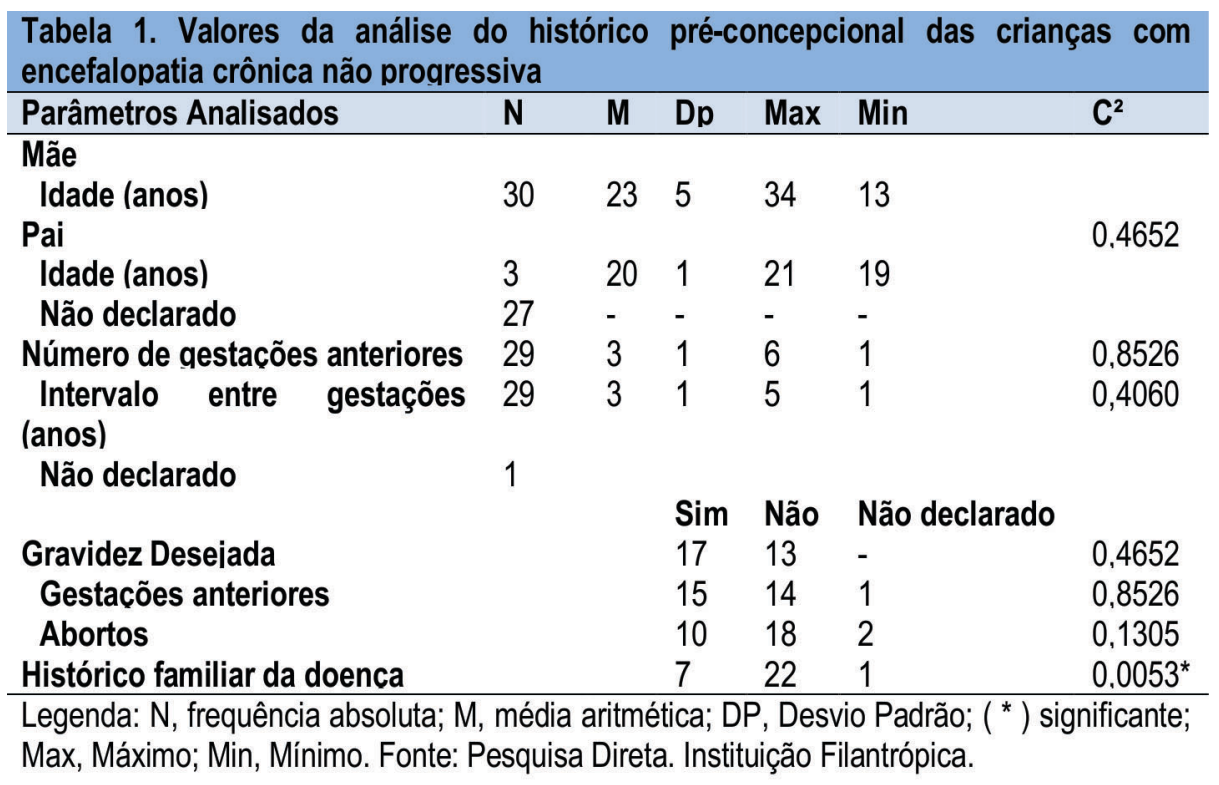

\begin{tabular}{|c|c|c|c|c|c|}
\hline \multirow{2}{*}{ DADOS } & \multicolumn{2}{|c|}{ Sim } & \multicolumn{2}{|c|}{ Não } & \multirow[t]{2}{*}{$\mathrm{C}^{2}$} \\
\hline & $\mathrm{n}$ & $\%$ & $\mathrm{n}$ & $\%$ & \\
\hline Utilizacão de medicamentos abortivos & 4 & $13.3 \%$ & 26 & $86.7 \%$ & \\
\hline Consumo de drogas ou ingestão de bebidas alcoólicas & 2 & $6.7 \%$ & 28 & $93.3 \%$ & - \\
\hline Tabaqismo & 3 & $10.0 \%$ & 27 & $90.0 \%$ & - \\
\hline Realização de cuidados pré-natais & 27 & $90,0 \%$ & 3 & $10,0 \%$ & - \\
\hline
\end{tabular}

Legenda: N, frequência absoluta; \%, frequência relativa; -, valores de Qui-quadrado (c²) não aplicável devido a ausência de correlação. Fonte: Pesquisa Direta. Instituição Filantrópica 


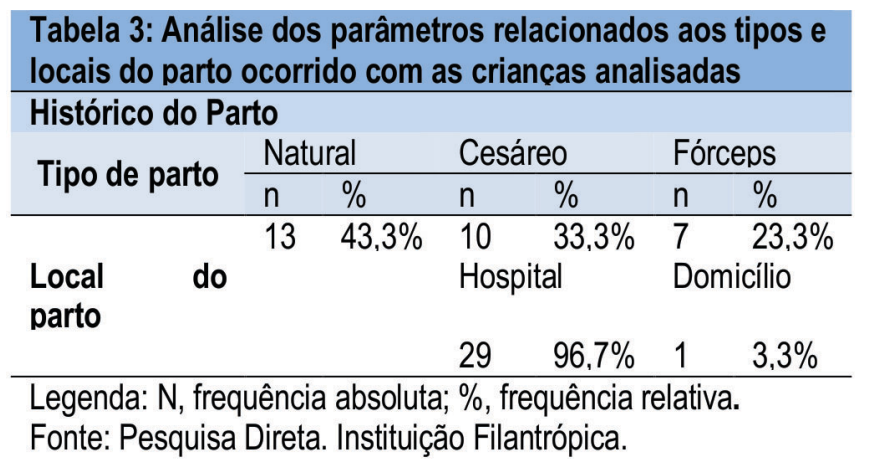

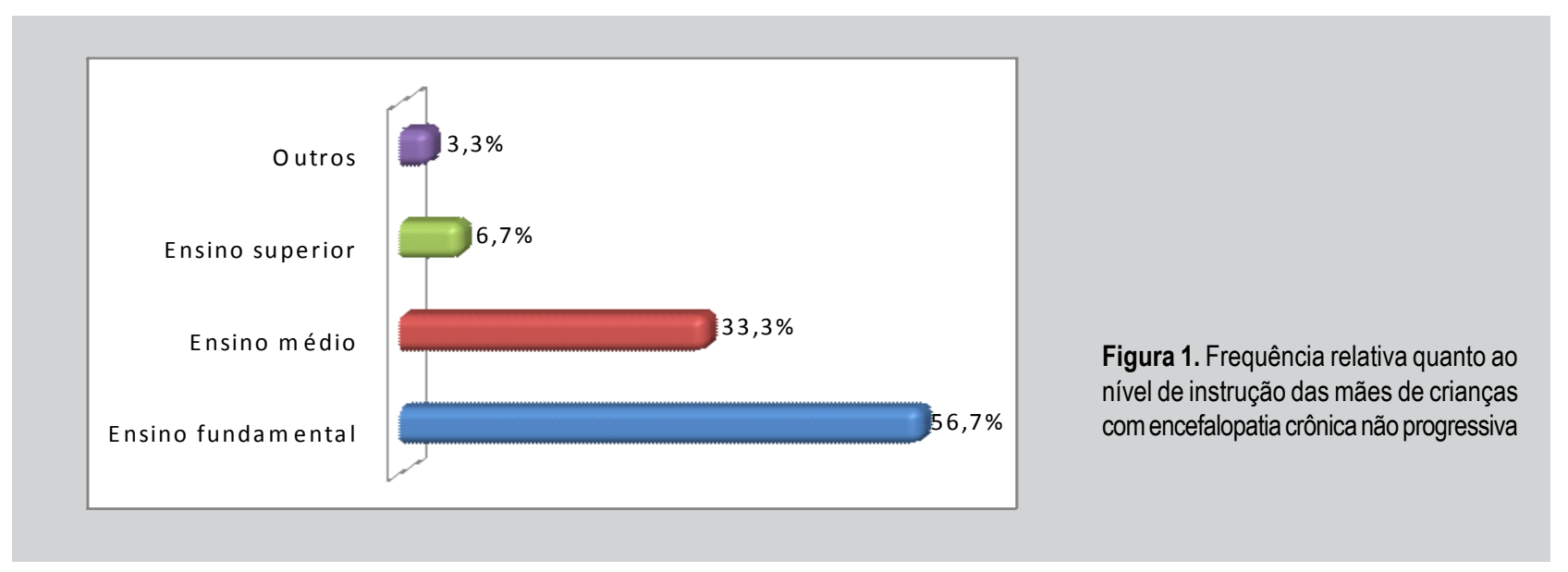

de Goiânia-GO ${ }^{23}$, em que foi observado uma proporção de $64,28 \%$ de crianças do gênero masculino. Outra pesquisa desenvolvida na Califórnia ${ }^{24}$, da qual participaram 25.545 crianças com ECNP, também evidenciou uma maior ocorrência de crianças do gênero masculino, correspondendo a $56 \%$ da amostra.

Deve-se considerar que a seleção da amostra deste estudo ocorreu por critérios de conveniência, com base nos pacientes presentes no momento do atendimento interdisciplinar, podendo assim não representar a real predominância do gênero masculino na ECNP, como cita a maioria dos estudos. E essa predominância de crianças do gênero masculino na maioria dos estudos observados sugere uma herança genética, devido a um traço recessivo ligado ao cromossomo $\mathrm{X}^{14}$.

Já em relação à idade média das crianças com ECNP, os achados de um delineamento transversal ${ }^{3}$, com crianças com ECNP atendidas no setor de odontologia da AACD em Recife-PE, a média das idades foi equivalente a 8,61 anos. Já uma outra pesquisa de corte transversal com abordagem quantitativa ${ }^{25}$, na região metropolitana do Vale do Aço, no Estado de Minas Gerais, com 49 crianças com ECNP, evidenciou que a maioria das crianças em atendimento encontrava-se na faixa etária entre três a sete anos e meio, assim os dados destes estudos concordam com os resultados encontrados na pesquisa atual.

Tais números são diretamente proporcionais ao aumento nos últimos 10 anos, na sobrevivência crescente de recém-nascidos prematuros e com extremo baixo peso, nos quais os distúrbios neurológicos são mais frequentes, tal fato pode ser justificado pelo desenvolvimento de tecnologias mais avançadas e aperfeiçoamento dos cuidados em assistência perinatal, e desta forma possibilitando o atendimento destas crianças.

Um estudo descritivo e qualitativo realizado na cidade de Fortaleza-CE ${ }^{7}$, com 12 mães de crianças com diagnóstico clínico de ECNP, identificou que $58,33 \%$ eram primigestas. E uma pesquisa avaliativa, descritiva e sistemática também na cidade de Fortaleza$\mathrm{CE}^{8}$, evidenciou $44,2 \%$ de mães primigestas, divergindo dos dados deste estudo no que se refere à ocorrência de gestações anteriores (Tabela 1). A maior ocorrência de gestações anteriores na presente pesquisa pode ser justificada pelo baixo nível socioeconômico destas mães, assim como pela falta de conhecimento acerca dos critérios de prevenção 9 . Contudo esta variável não foi estatisticamente significativa.

No que diz respeito à ocorrência de gravidez desejada, um delineamento do tipo transversal realizado 
no Rio Grande do Sul, com 2557 puérperas, $65 \%$ relataram que a gravidez foi desejada, percentual bem próximo à taxa descrita para o Equador $(62,7 \%)$ e para a América do Sul $(49,8 \%)^{9}$. Um estudo quantitativo, transversal, analítico realizado em dez Unidades Básicas de Saúde, na cidade de Maceió-AL ${ }^{10}$, obtiveram uma taxa de $65,7 \%$ para a ocorrência de gravidez desejada, sendo que $8,6 \%$ destas mães já haviam abortado. Um outro estudo transversal $^{11}$, no município de São Paulo com 225 gestantes, apresentou 12 mães $(5,33 \%)$ com relatos referentes à ocorrência de abortos.

Os achados dos estudos citados acima reforçam os resultados encontrados na presente pesquisa, no que se refere a um maior percentual de crianças provenientes de uma gravidez desejada e um menor percentual para a ocorrência de abortos prévios. De certa forma, deve-se considerar a relevância metodológica referente aos termos "desejada" e "planejada", uma vez que tais respostas podem variar de acordo com o estado psicológico da mãe, podendo sofrer alterações posteriores ${ }^{12}$. Assim, pode-se justificar a ausência de significância desta variável no desencadeamento da ECNP. Em contrapartida, a variável histórico familiar da doença foi significativa estatisticamente, constituindo-se como um fator de risco. Ratificando a questão da transmissão genética a partir de traço recessivo ligado ao cromossomo $\mathrm{X}^{14}$

Em uma outra pesquisa de corte transversal na cidade de Caxias do Sul-RS, com 239 mães, foi observado que $2,3 \%$ consumiram drogas ilícitas ${ }^{13}$. Um levantamento epidemiológico desenvolvido na Austrália, entre julho de 2008 e março de 2010 evidenciou a ocorrência de $14 \%$ de casos de tabagismo durante o período gestacional. Estes achados corroboram com os dados encontrados na presente pesquisa, referentes a proporções reduzidas de mães tabagistas e ainda em relação às que consumiram drogas ilícitas no período gestacional ${ }^{14}$.

O índice de consultas pré-natais também está dentro da média nacional, na qual pelo menos $90 \%$ das gestantes brasileiras iniciam pré-natal e cerca de $80 \%$ delas encontram-se imunizadas contra o tétano neonatal ${ }^{15}$. Esse percentual pode estar associado ao aumento das políticas de incentivo, divulgadas na mídia pelo governo federal.

O reduzido percentual de mulheres que ingeriram bebidas alcoólicas, consumiram drogas e utilizaram medicamentos abortivos durante o período gestacional, pode ser justificado pela grande quantidade de mães que procuraram assistência pré-natal. Supõese que, gestantes que recebem apoio são mais propensas a adotar hábitos saudáveis. Além da questão da aceitabilidade social, gestantes geralmente estão cientes de que o álcool é danoso ao feto e que estes hábitos são inaceitáveis pela sociedade ${ }^{16}$.
Um estudo desenvolvido com 239 mães constatou uma ocorrência de $59 \%$ de partos cesáreos ${ }^{17}$. De acordo com dados de um outro estudo ${ }^{18}$, realizado com 331 mães, no município de Sarandi-PR, 92,9\% dos partos ocorreram por via cesárea. Já resultados de um delineamento transversal realizado em uma maternidade de Campinas-SP, mostraram a ocorrência de $70 \%$ de partos naturais, sendo que $18,3 \%$ foram realizados com a aplicação de fórceps ${ }^{19}$.

Um delineamento transversal realizado na Universidade Federal do Rio Grande-RS com 2557 mães, ressalta que o uso de fórceps ocorreu em $7 \%$ da amostra $^{9}$. Dessa forma, percebe-se que estes dados concordam com a pesquisa atual com relação aos percentuais de ocorrência dos partos naturais e por fórceps e discordam quanto ao percentual de partos cesáreos.

As taxas reduzidas de partos por via cesárea encontrados neste estudo pode ter ocorrido provavelmente devido aos atuais incentivos aos partos naturais. A via vaginal é mais segura para a mãe e para o feto; benefícios advindos de partos naturais, são bem descritos na literatura, uma vez que as contrações ocorridas durante este procedimento, promovem a maturação pulmonar do feto, diminuindo a ocorrência de problemas respiratórios ${ }^{20}$.

Em uma análise referente às informações sobre o acesso geográfico ao parto hospitalar nos municípios brasileiros, da qual participaram 5564 municípios, os resultados revelaram que pouco mais de $70 \%$ dos municípios brasileiros possuem menos de $1 \%$ de partos realizados em ambiente domiciliar ${ }^{21}$. O controle da assistência ao parto pela medicina, trouxe uma mudança marcante, a transferência do ambiente domiciliar para o hospitalar ${ }^{22}$. Estes achados confirmam os percentuais reduzidos de partos realizados em ambiente domiciliar encontrados na pesquisa atual.

Um estudo transversal de caráter qualitativo e quantitativo realizado no complexo hospitalar da Santa Casa no Rio Grande do Sul, com 13 famílias, foi observado que as mães de crianças com diagnóstico de encefalopatia crônica não progressiva, em relação à escolaridade, $30,77 \%$ tinham o ensino fundamental incompleto, $15,38 \%$ da amostra haviam concluído o ensino fundamental e $53,85 \%$ possuíam ensino médio completo $^{26}$.

Após a realização de uma coleta de dados com 100 mulheres no Pará, quanto ao nível de escolaridade das mães investigadas, se obtiveram taxas de $41 \%$ de entrevistadas que relataram ter concluído o ensino médio, seguida de $20 \%$ que concluiu o ensino fundamental completo.

Os achados acima discordam dos resultados encontrados na presente pesquisa, sendo que um maior percentual de mães só havia estudado até o ensino 
fundamental. Os baixos índices de escolaridade dificultam o acesso às informações e às medidas de prevenção e promoção à saúde, fundamentais para a adequação da vida pessoal e reprodutiva. Nesse sentido, a baixa escolaridade, associada à falta de incentivos sociais, culminam em barreiras econômicas, impedindo o acesso aos melhores serviços de saúde, constituindo-se assim fatores de riscos importantes para ocorrência da $\mathrm{ECNP}^{27}$.

\section{CONCLUSÃO}

Os achados sugerem que os antecedentes socioeconômicos e gestacional das mães podem interferir direta ou indiretamente no desenvolvimento da ECNP. Assim, estes resultados podem contribuir para que haja uma melhoria das condições socioeconômicas desta população, uma vez que fornece subsídios para a formulação de políticas públicas que primem pela facilitação do acesso aos serviços de saúde e pela assistência de forma integral.

\section{REFERÊNCIAS}

1. Mello R, Ichisato SMT, Marcon SS. Percepção da família quanto à doença e ao cuidado fisioterapêutico de pessoas com paralisia cerebral. Rev Bras Enferm. 2012; 65(1): 104-109.

2. Oliveira AIA, Golin MO, Cunha MCB. Aplicabilidade do Sistema de Classificação da Função Motora Grossa (GMFCS) na paralisia cerebral - revisão da literatura. Arq Bras Ciên Saúde. 2010 35(3): 220-4

3. Lemos ACO, Katz CRT. Condições de saúde bucal e acesso ao tratamento odontológico de pacientes com paralisia cerebra atendidos em um centro de referência do Nordeste-Brasil. Revista CEFAC. 2012; 14(5): 861-871.

4. Cardoso JE, Barbosa RHS. O desencontro entre desejo e realidade: a "indústria" da cesariana entre mulheres de camadas médias no Rio de Janeiro. Physis: Rev de Saúde Coletiva. 2011; 22(1): 35 52.

5. Jodas DA, Scochi MJ, Moura MB, Tiwata MZ. Atendimento à criança e às mães: investigação do óbito evitável em menores de cinco anos. Rev Gaúcha de Enferm. 2011; 32(4): 669-75.

6. Gravena AAF Sassi A, Marcon SS, Peloso SM. Resultados perinatais nos extremos da vida reprodutiva e fatores associados ao baixo peso ao nascer. Rev Gaúcha de Enferm. 2011; 32(2) 352-358.

7. Gondim KM, Carvalho ZMF. Sentimentos das mães de crianças com paralisia cerebral à luz da teoria de Mishel. Escola Anna Nery. 2012; 16(1): 11-16.

8. Peixoto CR, Freitas LV, Teles LMR, Campos FC, Paula PF, Damasceno AKC. O pré-natal na atenção primária: o ponto de partida para a reorganização da assistência obstétrica. Rev de Enfermagem UERJ. 2011; 19(2): 286-291.

9. Cesar JA, Mendoza-Sassi RA, Gonzalez-Chica DA, Mano PS Características sociodemográficas e de assistência à gestação e ao parto no extremo sul do Brasil. Cad de Saúde Pública. 2011; 27(1): 985-994.

10. Correia DS, Calheiros AMN, Calvacante JC, Santos LVA, Vieira MJ. Adolescentes grávidas: sinais, sintomas, intercorrências e presença de estresse. Rev Gaúcha de Enferm. 2011; 32(1): 1-8.

11. Nomura RMY, Benute GRG, Azevedo GD, Dutra SEM, Borsari CG, Rebouças MSS et al. Depressão, aspectos emocionais e sociais na vivência do aborto: comparação entre duas capitais Brasileiras. Rev Assoc Med Bras. 2011; 57(6): 644-650.

12. Prietsch SOM, Gonzalez-Chica DA, Cesar JA, Mendoza-Sassi RA et al. Gravidez não planejada no extremo Sul do Brasil:prevalência e fatores associados. Cad Saúde Pública. 2011; 27(10): 19061916

13. Araújo BF, Zatti H, Filho PFO, Coelho MB, Olmi FB, Guaressi TB, Madi JMI. Influência do local de nascimento e do transporte sobre a morbimortalidade de recém-nascidos prematuros. J. Pediatr. 2011; 87(3): 257-262.

14. O' Callaghan ME, MacLennan AH, Gibson CS, McMichael GL, Haan EA, Broadbent JL et al. Epidemiologic associations with cerebral palsy. American College of obstetricians and gynecologists. 2011; 118(3): 576-82.
15. Mano OS, Cesar JA, Gonzalez-Chica DA, Neumann NA et al. Iniquidade na assistência à gestação e ao parto em município do semiárido brasileiro. Rev Bras Saude Mater Infant. 2011; 11(4): 381-388.

16. Silva I, Quevedo LA, Silva RA, Oliveira SS, Pinheiro RT. Associação entre abuso de álcool durante a gestação e o peso ao nascer. Rev de Saúde Pública. 2011; 45(5): 864-869.

17. Silva FA. Avaliação da dor em crianças com paralisia cerebral grave e relação entre características clínicas e demográficas. Trabalho de conclusão de curso: Porto Alegre: Universidade Federal do Rio Grande do Sul; 2012.

18. Sass A, Gravena AAF, Pelloso SM, Marcon SS. Resultados perinatais nos extremos da vida reprodutiva e fatores associados ao baixo peso ao nascer. Rev Gaúcha de Enferm. 2011; 32(2): 352-358.

19. Surita FGC, Suarez MBB, Siani S, Silva JLP. Fatores associados ao baixo peso ao nascimento entre adolescentes no Sudeste do Brasil. Rev Bras Ginecol Obstet. 2011; 33(10): 286-291.

20. Coelho MAL, Katz L, Coutinho I, Hofmann A, Miranda L, Amorim M. Perfil de mulheres admitidas em uma UTI obstétrica por causas não obstétricas. Rev Assoc Med Bras. 2012; 58(2): 160-167.

21. Almeida WS, Szwarcwald CL. Mortalidade infantil e acesso geográfico ao parto nos municípios brasileiros. Rev de Saúde Pública. 2012; 46(1): 68-76.

22. Raspantini PR. Rede de atenção ao nascimento e fatores de risco associados ao parto cesáreo em três regiões de saúde do Estado de São Paulo. Dissertação (Mestrado em Epidemiologia). São Paulo: Faculdade de Saúde Pública, Universidade de São Paulo; 2012.

23. Rézio GS, Cunha JOV, Formiga CKMR. Estudo da independência funcional, motricidade e inserção escolar de crianças com paralisia cerebral. Rev Bras de Educ Espec. 2012; 18(4): 601-614

24. Brooks J, Dia S, Shavelle R, Strauss D. Low weight,Morbidity, and Mortality in children with Cerebral palsy: New clinical Growth charts. Pediatrics. 2011; 128(2): 299-307.

25. Assis-Madeira EA, Carvalho SG, Blascovi-Assis SM. Desempenho funcional de crianças com paralisia cerebral de níveis socioeconômicos alto e baixo. Rev Paul de Pediatr. 2013; 31(1): $51-7$.

26. Susin FP et al. Perfil de pacientes com paralisia cerebral em uso de gastrostomia e efeito nos cuidadores. Revista CEFAC,São Paulo, v.14, n.5, p.933-942, set./out, 2012.

27. Souza LF, Botelho NM. Fatores de risco para parto prematuro em puérperas que tiveram parto pretermo. Revista para Médicos, vol. 25, n. 4. out./dez, 2012.

\section{Correspondência}

Luciana de Sousa Mata Ferreira

Endereço: Quadra 47, Casa 6, Setor A, Bairro Mocambinho I

Teresina - Piauí - Brasil

Email: luciana_mata@outlook.com 